\title{
Lumbar Spine Disc Herniation Diagnosis with a Joint Shape Model
}

\author{
Raja S Alomari ${ }^{1}$, Jason J Corso ${ }^{1}$ Vipin Chaudhary ${ }^{1}$, and Gurmeet Dhillon ${ }^{2}$ \\ 1 State University of New York at Buffalo, SUNY, Buffalo, NY, USA, \\ \{ralomari, jcorso, vipin\}@buffalo.edu, \\ WWW home page: http: //cadi.buffalo.edu \\ 2 Proscan Radiology Buffalo, Buffalo, NY, USA, \\ gdhilloneproscan.com,
}

\begin{abstract}
Lower Back Pain (LBP) is the second most common neurological ailment in the United States after the headache. It costs over \$100 Billion annually in treatment and related rehabilitation costs including worker compensation. In fact, it is the most common reason for lost wages and missed work days. Degenerative Disc Disease (DDD) is the major abnormality that causes LBP. Moreover, Magnetic Resonance Imaging (MRI) test is the main clinically approved noninvasive imaging modality for the diagnosis of DDD. However, there is over $50 \%$ inter- and intra-observer variability in the MRI interpretation that urges the need for standardized mechanisms in MRI interpretation. In this paper, we propose a Computer Aided Diagnosis (CAD) System for Disc Degenerative Disease detection from clinical Magnetic Resonance Imaging (MRI). This CAD produces a reproducible and clinically accurate diagnosis of the DDD for lumbar spine. We design a classifier to automatically detect degenerated disc (also clinically known as Herniation) using shape potentials. We extract these shape potentials by jointly applying an active shape model (ASM) and a gradient vector flow snake model (GVF-snake). The ASM roughly segments the disc by the detection of a certain point distribution around the disc. Then, we use this point distribution to initialize a GVF-snake model to delineate the posterior disc segment. We then extract the set of shape potentials for our Gibbs-based classifier. The whole work flow is fully automated given the full clinical MRI. We validate our model on 65 clinical MRI cases (6 discs each) and achieve an average of $93.9 \%$ classification accuracy. Our shape-based classifier is superior in classification accuracy compared to the state-of-the-art work on this problem that reports $86 \%$ and $91 \%$ on 34 and 33 cases, respectively.
\end{abstract}

Keywords: Lumbar Spine Diagnosis, MRI, Disc Degenerative Disease

\section{Introduction}

Low Back Pain has a major economic impact in the United States with over \$100 Billion annually in related treatment and rehabilitation costs [1]. It is the most common reason why patients visit a physician office besides the common cold. In fact, it is the most common reason patients visited the emergency room in the U.S. in 2008. There were over 3.4 Million emergency rooms visits, an average of 9400 visits a day, specifically 
for Low Back Pain [2]. Low back Pain has high societal impact as it disrupts individuals lives impacting over $80 \%$ of people [3]. Moreover, it is the most common reason behind job-related disability and is the second most common neurological ailment after headache [3]. It is a prominent chronic disease that causes major disruption in people's lives.

Nevertheless, the diagnostic decision is highly subjective and relies on two major factors: the radiologist's diagnostic report and the neurological exam findings. The most common current clinically approved standard for Low Back Pain diagnosis is the Magnetic Resonance Imaging (MRI) procedure. However, individual radiologists interpreting clinical Magnetic Resonance Imaging (MRI) studies are highly subjective with over 50\% inter-observer variation [4]. This high inter-radiologist variation significantly influences therapeutic treatment, medical insurance decision makers, and judiciary personnel decisions. On the other hand, the clinical diagnosis is highly variable that nothing certain can be said regarding the clinical diagnosis of Low Back Pain [5]. Providing a reproducible computerized MRI interpretation may reduce the existing variability, and hence, standardize the diagnostic decisions that lead to reduced costs on unnecessary treatment.

Surprisingly, there is no CAD system for the lumbar spine that yet has clinical applicability. We are building our system motivated by the clinical practice of lumbar diagnosis. In this paper, we propose a reliable, robust, and accurate diagnosis for disc herniation which is the main condition that causes failed low back syndrome. We, however,

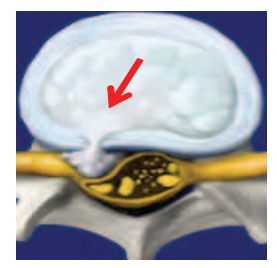

(a) Axial Model

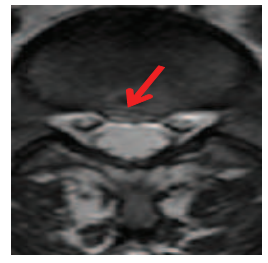

(b) Axial MRI

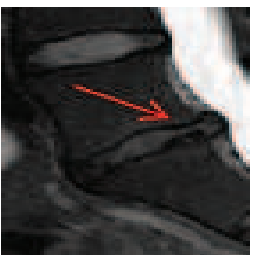

(c) Sagittal MRI

Fig. 1. (a) A right-sided disc herniation illustrative model [6]. (b) Axial view (bottom-up) MRI of a right-sided disc herniation from our data. (c) Corresponding sagittal view of the herniated disc from our dataset.

point out that the nomenclature has been a controversial issue in spine diseases which is outside the scope of this paper. We target the problem of the leak of the nucleus pulposus (as shown in Fig. 1) that causes pressure on the nerve root resulting in the pain and numbness to the patient where the pain, most of the time, irritates to the knees causing major disruption of the patients life. We use the nomenclature of Fardon et al [7] that has been endorsed by the major American and European radiologists associations including ASSR, ASNR, AANS, CNS, ESNR, and many others. For the rest of this paper, we call this condition as Herniation.

Disc herniation always occurs in the posterior segment of the disc. The inner gellike material of the disc, nucleus pulposus, leaks out pressing on a nerve root through a tear in the fibrous wall of the disc, annulus fibrosus [8], as illustrated in Fig. 1, where we show an axial illustrative model and a corresponding clinical MRI (from our dataset) for a right-sided disc herniation with both the axial and sagittal views. 
Shape of the posterior segment of the disc, from the sagittal view, is the primary diagnostic tool for the radiologist. The axial view is used for confirmation and for quantification. Working in the sagittal view, our method extracts information of the posterior segment of the disc in a two-step process. First, we use an active shape model to roughly localize a point distribution for the disc body. Then, we have a GVF-snake to delineate the posterior segment of the disc using the outcome of the ASM as its initialization. Because the ASM is a linear model and captures Gaussian point distributions, we add the GVF-snake step to delineate the non-linear shape of the disc posterior segment which is the main technical innovation in this paper. We validate our method on a clinical dataset of sixty-five cases and achieve over 93\% average classification accuracy.

We also compare our results to the most recent work on disc herniation diagnosis by Alomari et al $[9,10]$ that jointly model shape and intensity and we substantially outperform their results. Moreover, our shape-based classifier outperforms the recent work of Michopoulou et al [11] which is based on an intensity-based classifier. Both recent works test on 33 and 34 cases with an average herniation detection accuracy of $91 \%$ and $88 \%$, respectively. We validate our model on substantially variable dataset of 65 cases and achieve better accuracy over $93 \%$. Many researchers have proposed methods for the diagnosis of certain vertebral column abnormalities. Bounds et al [12] utilized a neural network for the diagnosis of back pain and sciatica. Sciatica might be caused by lumbar disc Herniation as well as many other reasons. They have three groups of doctors to perform diagnosis as their validation mechanism. They claimed a better accuracy than the doctors in the diagnosis. However, the lack of data prohibited them from full validation of their system. Similarly, Vaughn [13] conducted a research study on using neural network for assisting orthopedic surgeons in the diagnosis of lower back pain. They classified LBP into three broad clinical categories: Simple Low Back Pain (SLBP), Root Pain (ROOTP), and Abnormal Illness Behavior (AIB) and about 200 cases were collected over the period of 2 years with diagnosis from radiologists. They used 25 features to train the Neural Network (NN) including symptoms clinical assessment results. The NN achieved $99 \%$ of training accuracy and $78.5 \%$ of testing accuracy. This clearly shows training data overfitting.

Tsai et al [14] used geometrical features (shape, size and location) to diagnose herniation from 3D MRI and CT axial (transverse sections) volumes of the discs. In contrast, we do not presume the availability of the full volume axial view as it is not a clinical standard. They patented their work as a visualization tool for educational purposes. Recently, Michopoulou et al [11] applied three variations of fuzzy c-means (FCM) to perform atlas-based disc segmentation. Then, they used this segmentation for classification of the disc as either a normal or degenerative disc. They used an intensity-based Bayesian classifier and achieved 86\%-88\% classification accuracy on 34 cases (five discs each) based on their semi-automatic segmentation of the disc. Similarly, Alomari et al $[9,10]$ proposed utilizing a shape and an intensity-based classifier that utilizes an active shape model to extract the shape potentials. However, because the ASM cannot capture the non-linearly shaped posterior segment of the herniated disc, they achieved about $91 \%$ on 33 clinical cases. We extend both these works and present our technical novelty by concentrating on the posterior segment of the disc and capturing that with an additional GVF-snake model on top of the ASM. Furthermore, we reduce the effect 
of intensity-based information due to the signal intensity inhomogeneity with clinical MRI. We also significantly add variability in the dataset by validating our joint model on 65 clinical cases as opposed to 33 and 34 cases. Furthermore, we achieved an average of $93 \%$ accuracy which substantially outperforms both state-of-the-art results given the dataset size difference.

\section{Proposed Method}

Our approach has four steps: Disc Localization, Disc Segmentation, Herniation Delineation, and Herniation Classification. This section explains each step:

Disc Localization: The system automatically locates the middle sagittal slice from the MRI volume by index. Then our automatic method starts by a localization step that provides a point inside each disc using the two-level probabilistic model proposed by Corso et al $[15,16]$. Their model labels the set of discs with high level labels $\mathrm{D}=\left\{d_{1}, d_{2}, \ldots, d_{6}\right\}$ where each $d_{i}=\left(x_{i}, y_{i}\right)^{\top}$ is the coordinates of the disc point (some point in the disc). They solve the optimization problem:

$$
\mathrm{D}^{*}=\arg \max _{\mathrm{D}} \sum_{\mathrm{L}} P(\mathrm{~L}, \mathrm{D} \mid \mathrm{I})=\arg \max _{\mathrm{D}} \sum_{\mathrm{L}} P(\mathrm{~L} \mid \mathrm{D}, \mathrm{I}) P(\mathrm{D})
$$

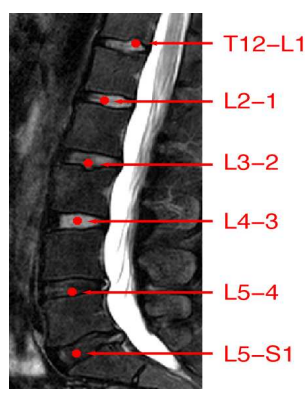

Fig. 2. Labeling lumbar discs in a sagittal T2weighted MRI $[15,16]$.

where $\mathrm{L}=\left\{l_{i}, \forall i \in \mathrm{L}\right\}$ is a set of auxiliary variables, called disc-label variables that are introduced to infer $D$ from the sagittal image. Each disc-label variable can take a value of $\{-1,+1\}$ for non-disc or disc, respectively. The disc-labels make it plausible to separate the disc variables from the image intensities, i.e., the disc-label L variables capture the local pixel-level intensity models while the disc variables D capture the high-level geometric and contextual models of the full set of discs. The optimization is solved with a generalized expectation minimization (gEM) algorithm [15, 16]. Fig. 2 shows a lumbar sagittal view with labeled discs. Then we obtain a fixed window of $60 \times 120$ pixels around each point. This sub-image size is enough to provide the whole disc region for each of the discs connected to the five lumbar vertebrae as shown in Fig. 2.

Disc Segmentation: We use an active shape model [17] for roughly segmenting the disc body boundary. This step finds the rough shape of the disc body regardless of the herniated (posterior) part. To prepare the training data, we manually select the image slice where herniation is most obvious. Then, we manually mark nine landmark points according to the map shown in Fig. 3. Specifying these landmarks locations is only based on our expertise in the disc segmentation. We name these landmark points from $\mathrm{k}_{1}$ to $\mathrm{k}_{9}$. Similar to [17], we initially calculate the mean shape $\bar{x}=\frac{1}{N} \sum_{1}^{N} x$ where $N$ is the size of the training data. Then each disc shape $x_{i}$, where $i \in\{1, \ldots, N\}$, is recursively aligned to the mean shape $\bar{x}$ using generalized Procrustes Analysis to remove translational, rotational, and isotropic scaling from the shape. 


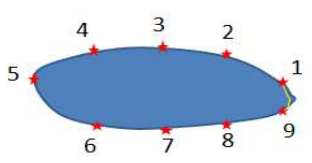

(a) Normal Model

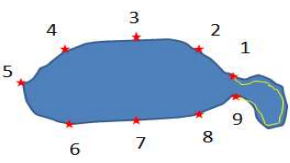

(b) Herniated Model

Fig. 3. Illustrative Model [sagittal view] for (a) clinically normal disc (b) herniated disc showing the point distribution $\left(\mathrm{k}_{1}-\mathrm{k}_{9}\right)$ as well as a contour (yellow) that delineates the edge map between points $\mathrm{k}_{1}$ and $\mathrm{k}_{9}$. This figure shows the irregular shape of the normal disc.

Then, we model the remaining variance around the mean shape with principal components analysis (PCA) to extract the eigenvectors of the covariance matrix associated with $98 \%$ of the remaining point position variance according to the standard method for deriving the ASM's linear shape representation.

However, we do not use the original MRI image for training the ASM. Rather, we utilize a feature image I that enhances the disc shape by emphasizing the boundaries of the disc and the Thecal Sac (the extension of the spinal canal at the lumbar level [8]). We produce I by applying a range filter $R$ on the pixelwise addition of the normalized coregistered T1- and T2-weighted protocols of the sagittal images $\mathrm{I}=$

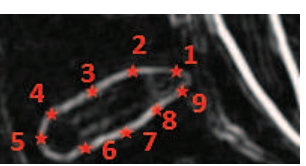

(a) Normal

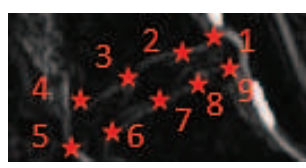

(b) Herniated

Fig. 4. Feature image result of the range filter $\mathrm{R}$ for (a) Normal disc (b) Herniated disc. The ASM point distribution is shown according to the map in Fig 3.

$\mathrm{R}(\mathrm{T} 1+\mathrm{T} 2)$ where $\mathrm{T} 1$ and $\mathrm{T} 2$ are the normalized T1- and T2-weighted MRI images for the same case. These two images are manually co-registered during the acquisition of the MRI in the clinical standard. $R$ is the range filter operator where the intensity levels in each $3 \times 3$ window are replaced by the range value (maximum - minimum) in that window. This operator $R$ has high values in abrupt-change regions and small values in smooth regions. Fig. 4 shows the features images I for a normal- and a herniated-disc. The ASM landmark points are also shown in the figure to clarify the ASM land-marking step.

To apply ASM for detection of the point distribution of the disc body boundary, we apply the mean shape $\bar{x}$ around the disc point produced by the localization step. Then, we allow the ASM to converge and obtain the boundary.

We apply the GVF-snake by initializing its contour (to the line connecting the two points $k_{1}$ and $k_{9}$ ). Figure 5 show two examples of the convergence of the GVF-snake for both a normal disc (Fig. 5(a)) and a herniated one (Fig. 5(b)). The figure also shows the normalized gradient vector field for the sub-image as well as a zoomed GVF field for the area of interest (posterior part of the disc).

Herniation Delineation: The ASM segmentation of the disc cannot capture the inherent variations produced by the disc herniation at the posterior segment of the disc. Furthermore, we seek for a single model for the disc regardless whether it is herniated 
or not. Thus, we use an active contour to delineate the posterior segment of the disc. We select the GVF-snake proposed by Xu and Prince [18] because it has been proved to move toward desired image properties such as edges including concavities. GVF-snake is the parametric curve that solves:

$$
\mathbf{x}_{t}(s, t)=\alpha \mathbf{x}^{\prime \prime}(s, t)-\beta \mathbf{x}^{\prime \prime \prime \prime}(s, t)+\mathbf{v}
$$

where $\alpha$ and $\beta$ are weighting parameters that control the contour's tension and rigidity, respectively. $x^{\prime \prime}$ and $x^{\prime \prime \prime \prime}$ are the second and fourth derivatives, respectively, of $x$. $\mathbf{v}(x, y)$ is the gradient vector flow (GVF), $s \in[0,1]$, and $t$ is time component to make a dynamic snake curve from $x(s)$ yielding $x(s, t)$.

GVF-snake requires an edge map that is a binary image highlighting the desired features (edges) of the image. Most researchers use Canny edge detector or Sobel operator on the original image such as [19] for liver segmentation. We present the GVF-snake with a canny edge map applied on our feature image I.

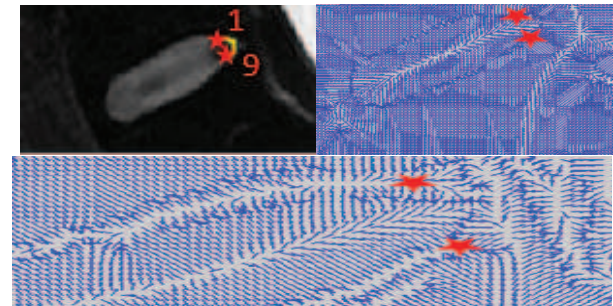

(a) Normal

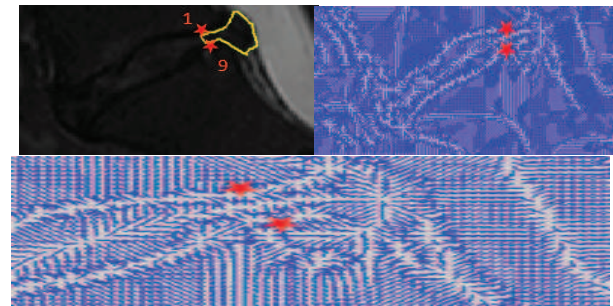

(b) Abnormal

Fig. 5. (Top-left) Shows the resulting GVF-contour for (a) normal (b) abnormal, on T2-weighted image. (Top-right) The corresponding normalized GVF field showing the two initialization landmarks $\mathrm{k}_{1}$ and $\mathrm{k}_{9}$. (Bottom) A zoomed version of the GVF field to clearly show the vectors.

Herniation Classification: We design a binary Bayesian classifier:

$$
n^{*}=\arg \max _{n} P(n \mid \mathrm{S})
$$

where $n$ is a binary random variable stating whether it is a herniated or a normal disc, $\mathrm{S}$ incorporates shape features extracted from both the GVF-snake and the ASM convergence. We utilize a Gibbs distribution with two shape potentials:

$$
P(n \mid \mathrm{S})=\frac{1}{Z[n]} \exp ^{-\left[\alpha_{1} U_{\mathrm{S} 1}+\alpha_{2} U_{\mathrm{S} 2}\right]}
$$

where S represents the shape features extracted from both the ASM convergence and the GVF-snake, $Z[n]$ is the normalization factor of the Gibbs distribution, $\alpha_{1}$ and $\alpha_{2}$ are tuning parameters. We define two shape potentials: 1) $U_{\mathrm{S} 1}$ models the GVF-snake delineation for the posterior segment of the disc. 2) $U_{\mathrm{S} 2}$ models the major axis of the ASM converged disc shape. 
Table 1: Cross Validation Results: Each row tests randomly selected 35 cases.

\begin{tabular}{|c|ccc|ccc|c|}
\hline Set & L5-S1 & L4-5 L3-4 & L2-3 & L1-2 & T12-L1 & Accuracy \\
\hline 1 & 32 & 32 & 34 & 34 & 35 & 34 & $95.7 \%$ \\
2 & 33 & 32 & 32 & 31 & 34 & 35 & $93.8 \%$ \\
3 & 33 & 34 & 32 & 33 & 33 & 34 & $94.8 \%$ \\
4 & 31 & 30 & 32 & 33 & 33 & 34 & $91.9 \%$ \\
5 & 31 & 32 & 32 & 33 & 34 & 33 & $92.9 \%$ \\
\hline 6 & 33 & 32 & 32 & 31 & 32 & 33 & $91.9 \%$ \\
7 & 33 & 32 & 34 & 34 & 33 & 33 & $94.8 \%$ \\
8 & 30 & 31 & 32 & 31 & 34 & 33 & $91.0 \%$ \\
9 & 30 & 33 & 34 & 34 & 35 & 35 & $95.7 \%$ \\
10 & 32 & 33 & 34 & 34 & 34 & 35 & $96.2 \%$ \\
\hline$(\%)$ & 90.9 & 91.7 & 93.7 & 93.7 & 96.3 & 96.9 & - \\
\hline \multicolumn{7}{|c|}{ Average Accuracy } & $\mathbf{9 3 . 9 \%}$ \\
\hline
\end{tabular}

Table 2: Calculation of specificity (96.6\%) and sensitivity (86.4\%).

\begin{tabular}{|c|c|c|}
\hline & \multicolumn{2}{|c|}{ Gold standard } \\
\hline & Herniated & Normal \\
\hline Herniated & $170(\mathrm{TP})$ & $53(\mathrm{FP})$ \\
\hline Normal & $25(\mathrm{FN})$ & $652(\mathrm{TN})$ \\
\hline
\end{tabular}

We extract the first shape potential $U_{\mathrm{S} 1}$ from the GVF-snake delineation of the posterior disc segment. The longer the contour, the more likely it delineates a herniated segment as shown in Fig. 5 by the yellow line between the points $k_{1}$ and $k_{9}$. To capture the length of the GVF-snake contour, we model the number of points that are sampled by the final GVF-contour. The GVF-snake interpolates the pixels by having a maximum of two pixels between each point. Thus, we define:

$$
U_{\mathrm{S} 1}=\frac{\left(e_{1}-\mu_{e_{1}}\right)^{2}}{2 \sigma_{e_{1}}^{2}}
$$

where $e_{1}$ is the number of interpolated points along the delineated GVF contour, $\mu_{e_{1}}$ $\sigma_{e_{1}}^{2}$ are the expected and the variance of the interpolated points on the GVF-contour, respectively. We estimate both $\mu_{e_{1}}$ and $\sigma_{e_{1}}^{2}$ from the training data.

The secondary shape potential, $U_{\mathrm{S} 2}$, is motivated by the clinical observation that the herniated disc collapses due to the leak of the nucleus pulposus causing average lengthening in the major axis of the disc as shown in Fig. 1. We utilize this by incorporating this second shape potential $U_{\mathrm{S} 2}$ :

$$
U_{\mathrm{S} 2}=\frac{\left(e_{2}-\mu_{e_{2}}\right)^{2}}{2 \sigma_{e_{2}}^{2}}
$$

where $e_{2}$ is the disc major axis length, $\mu_{e_{2}}$ is the expected major axis length of the disc, $\sigma_{e_{2}}^{2}$ is the variance of the major axis length of the disc. We learn both $\mu_{e_{2}}$ and $\sigma_{e_{2}}^{2}$ from the training data. We define $e_{2}$ by:

$$
e_{2}=\left|\frac{\mathrm{k}_{1}+\mathrm{k}_{9}}{2}-\mathrm{k}_{5}\right|_{2}
$$

where $\mathrm{k}_{1}, \mathrm{k}_{5}$, and $\mathrm{k}_{9}$ are the location coordinates of points 1,5 , and 9 , respectively, as shown in Fig. 4. The distance $e_{2}$ roughly measures the major disc axis length subtracting the average location of the right end points $\mathrm{k}_{1}$ and $\mathrm{k}_{9}$ and the left end point $\mathrm{k}_{5}$. 


\section{Data and Results}

Our clinical MRI dataset is captured by a Philips 3-Tesla scanner according to the clinical standard. Each case contains manually co-registered two sagittal views (T1- and T2weighted) as well as six axial T1-weighted slices for each disc. We use the clinical diagnosis reports to obtain our diagnosis gold standard. We validate our proposed method on 65 subjects with ages of 23 to 76 years old and with various types of abnormalities. We perform a cross-validation experiment where we leave 35 cases for testing and use the remaining 30 for training. We perform 10 rounds and each time, we randomly select the training and testing cases. We define the accuracy in each round (row in the Table 1) as the sum of correctly classified discs Accuracy $i=\left(1-\frac{1}{M} \sum_{j=1}^{K}\left|g_{i j}-n_{i j}\right|\right) * 100 \%$ where $i$ is the lumbar disc level, $1 \leq i \leq 6, M$ is the testing set size in each round (35 cases).

Table 1 shows the classification results from the cross validation experiment. We achieve an average of $93.9 \%$ accuracy on disc diagnosis. Each row in the table represents one round of the cross-validation. Thus, it represents 35 cases with 6 discs each case. We show the number of correctly classified discs at each disc level (column) out of 35 discs. We further compute the overall specificity and sensitivity where:

$$
\begin{aligned}
\text { Specificity } & =\frac{T N}{T N+F P} \\
\text { Sensitivity } & =\frac{T P}{T P+F N}
\end{aligned}
$$

where FP is the number of false positives (normal discs diagnosed as herniated), TP is the number of true positives (correctly diagnosed herniated discs), $\mathrm{FN}$ is the number of false negatives (misclassified herniated discs), and TN is the number of true negatives (correctly classified normal discs). Table 2 shows another cross validation experiment with 15 randomly selected cases for 10 rounds. This makes $15 \times 6$ (discs) x 10 (rounds)= 900 discs total (including repetitions). Within this cross validation experiment, there is a total of 78 misclassified discs: 25 herniated (false negatives) and 53 normal (false positives) as shown in Table 2 . We archive an overall specificity over $92 \%$ and sensitivity over $87 \%$.

Fig. 6 shows four examples from our dataset. It shows the convergence of the ASM point distribution (red dots and the linear connections) as well as the GVF-snake delineation (yellow curve). On the other hand, we compare our classification results to a Bayesian classifier that only models the disc appearance to show the effectiveness of modeling the shape. We run the same experiment with the same cases of Table 1 and obtain around $80 \%$ average classification accuracy. We justify that by the fact that despite Herniated discs produce lower intensity levels; in general, the difference in intensity with the normal disc is not enough to classify herniated and normal discs. However, a Bayesian intensity-based classifier can be useful for other diseases such as disc desiccation [20]. Fig. 6(c) shows a sample Herniated disc, with high intensity value, that was misclassified by the intensity-based classifier but correctly classified with our shapebased classifier. 


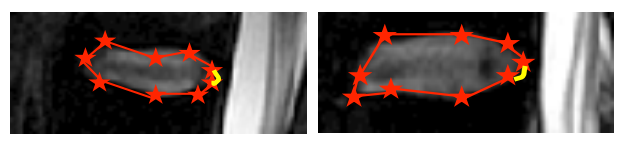

(a) Normal

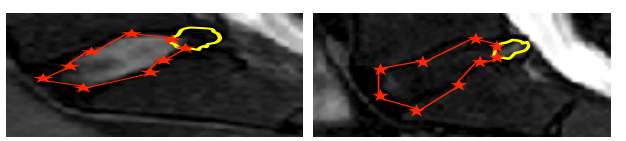

(b) Herniated (Left) high intensity, (Right) Low intensity

Fig. 6. Resulting ASM convergence and GVF-snake delineation for two normal cases and two abnormal ones.

\section{Conclusion}

We proposed a method for herniation diagnosis from lumbar area clinical MRI. We utilize a coordinated active shape and a gradient vector flow active contour models to extract shape features for detection of herniation. We use a Bayesian classifier and utilize a Gibbs-based distribution with shape potentials. We validate our method on a set of sixty five clinical MRI cases. We achieve an average of $93.9 \%$ classification accuracy with specificity $96.6 \%$ and sensitivity of $86.4 \%$. We also compared our results with the two state-of-the-art work and substantially outperform both of them due to our features that encompass the benefits of both works into a more robust classification model.

\section{References}

1. Crow, W.T., Willis, D.R.: Estimating cost of care for patients with acute low back pain: A retrospective review of patient records. Journal of the American Osteopathic Association 109(4) (April 2009) 229-233

2. Nelson, J., O'Neil, C., Richardson, C.J.: Treatment of low back pain: Exploring the costs. Health and Wellness (2012)

3. NINDS: National institute of neurological disorders and stroke (ninds): Low back pain fact sheet. NIND brochure (2008)

4. van Rijn, J.C., Klemets, N., Reitsma, J.B., Majoie, C.B.L.M., Hulsmans, F.J., Peul, W.C., Stam, J., Bossuyt, P.M., den Heeten, G.J.: Observer variation in mri evaluation of patients suspected of lumbar disk herniation. AJR. American journal of roentgenology (1) (Jan 2005)

5. Atlas, S.J., Deyo, R.A.: Evaluating and managing acute low back pain in the primary care setting. J Gen Intern Med 16(2) (Feb 2011) 120-131

6. Swarm: Interactive incorporation (viewmedica) - patient educatuion system. (2007)

7. Fardon, D.F., Milette, P.C.: Nomenclature and classification of lumbar disc pathology. SPINE 26(5) (2001) E93-E113

8. Snell, R.S.: Clinical Anatomy by Regions. 8th edn. Lipp. Will. and Wilkins (2007)

9. Alomari, R.S., Corso, J.J., Chaudhary, V., Dhillon, G.: Toward a clinical lumbar cad: herniation diagnosis. International Journal of Computer Assisted Radiology and Surgery 6 (2011) $119-126$ 
10. Alomari, R.S., Corso, J.J., Chaudhary, V., Dhillon, G.: Automatic diagnosis of lumbar disc herniation with shape and appearance features from mri. In: Proceedings of SPIE Conference on Medical Imaging (SPIE). (2010)

11. Michopoulou, S., Costaridou, L., Panagiotopoulos, E., Speller, R., Panayiotakis, G., ToddPokropek, A.: Atlas-based segmentation of degenerated lumbar intervertebral discs from $\mathrm{mr}$ images of the spine. IEEE Trans. on Biomedical Imaging 56(9) (Sept 2009) 2225-2231

12. Bounds, D., Lloyd, P., Mathew, B., Waddell, G.: A multilayer perceptron network for the diagnosis of low back pain. In: Proceedings of IEEE International Conference on Neural Networks. Volume 2., San Diego, CA (July 1988) 481-489

13. Vaughn, M.: Using an artificial neural network to assist orthopaedic surgeons in the diagnosis of low back pain. http://www.marilyn-vaughn.co.uk/lbpainresearchstudy.htm (june 2000)

14. Tsai, M.D., Jou, S.B., Hsieh, M.S.: A new method for lumbar herniated inter-vertebral disc diagnosis based on image analysis of transverse sections. Computerized Medical Imaging and Graphics 26(6) (2002) 369-380

15. Alomari, R.S., Corso, J.J., Chaudhary, V.: Labeling of lumbar discs using both pixel- and object-level features with a two-level probabilistic model. Medical Imaging, IEEE Transactions on 30(1) (jan. 2011) $1-10$

16. Corso, J.J., Alomari, R.S., Chaudhary, V.: Lumbar disc localization and labeling with a probabilistic model on both pixel and object features. In: Proceedings of Medical Image Computing and Computer Aided Intervention (MICCAI). Volume 5241 of LNCS Part 1., Springer (2008) 202-210

17. Cootes, T.F., Taylor, C.J.: Statistical models of appearance for medical image analysis and computer vision. In: Proceedings of SPIE Conference on Medical Imaging (SPIE). (2001) 236-248

18. Xu, C., Prince, J.L. In: Handbook of Medical Imaging. Academic Press, Baltimore, MD (2000)

19. Liu, F., Zhao, B., Kijewski, P., Wang, L., Schwartz, L.: Liver segmentation for ct images using gvf snake. Medical Physics 32(12) (Dec 2005) 3699-3706

20. Alomari, R.S., Corso, J.J., Chaudhary, V., Dhillon, G.: Desiccation diagnosis in lumbar discs from clinical mri with a probabilistic model. In: Proceedings of IEEE International Symposium on Biomedical Imaging (ISBI). (2009) 546-549 